\title{
DISCRETE CONVEX FUNCTIONS AND PROOF OF THE SIX CIRCLE CONJECTURE OF FEJES TÓTH
}

\author{
IMRE BÁRÁNY, ZOLTÁN FÜREDI AND JÁNOS PACH
}

1. Introduction, results. A system $\mathscr{C}$ of openly disjoint discs in the plane is said to form a 6-neighboured circle packing if every $C \in \mathscr{C}$ is tangent to at least 6 other elements of $\mathscr{C}$. (It is evident that such a system consists of infinitely many discs.) The simplest example is the regular circle packing all of whose circles are of the same size and have exactly 6 neighbours. L. Fejes Tóth conjectured that the regular circle packing has the interesting extremal property that, if we slightly "perturb" it, then there will necessarily occur either arbitrarily small or arbitrarily large circles. More precisely, he asked whether or not the following "zero or one law" (cf. [3], [6] ) is valid: If $\mathscr{C}$ is a 6-neighboured circle packing, then

$$
\frac{\text { inf } r(C)}{\sup r(C)}=\left\{\begin{array}{l}
1 \text { if } \mathscr{C} \text { is regular } \\
0 \text { otherwise }
\end{array}\right.
$$

where $r(C)$ denotes the radius of circle $C$, inf and sup are taken over all $C \in \mathscr{C}$.

We shall prove this conjecture in the following stronger form:

THEOREM 1. If $\mathscr{C}$ is a 6-neighboured circle packing which is not regular, then $\inf _{C \in \mathscr{C}} r(C)=0$.

Given a circle packing $\mathscr{C}$, we define a graph $G_{\mathscr{C}}=\left(V_{\mathscr{C}}, E_{\mathscr{C}}\right)$ on the vertex set $V_{\mathscr{C}}:=\mathscr{C}$ as follows: $C, C^{\prime} \in \mathscr{C}$ are joined by an edge (i.e., $C C^{\prime} \in E_{\mathscr{C}}$ ) if and only if $C$ and $C^{\prime}$ are tangent.

Now let $G=(V, E)$ be an arbitrary graph. For any $x \in V$, let $\Gamma_{G}(x)$ denote the set of all neighbours of $x$. That is,

$$
\Gamma_{G}(x)=\{y \in V \mid \quad x y \in E\} .
$$

A function $f: V \rightarrow \mathbf{R}$ is called convex (or subharmonic) on $G$ if

$$
\frac{1}{\left|\Gamma_{G}(x)\right|} \sum_{y \in \Gamma_{G}(x)} f(y) \geqq f(x) \quad \text { for every } x \in V .
$$

Any convex function defined on a connected finite graph is obviously constant. It is somewhat more surprising (but still easily seen) that every convex function on e.g. the rectangular lattice in the plane has either arbitrarily large positive values, or it is again constant (see [2]). This

Received June 7, 1983. 
observation inspired our proof of Theorem 1, though we will not rely upon it in what follows.

Our next theorem states that if $\mathscr{C}$ is a 6-neighboured circle packing then the function $r^{-1}(C),(C \in \mathscr{C})$ is convex on the graph $G_{\mathscr{C}}$ defined above.

THEOREM 2. Let $C$ be a disc of radius $r(C)$ which is tangent to at least 6 non-overlapping discs $C_{1}, \ldots, C_{k}$, $(k \geqq 6)$. Then

$$
\frac{1}{k} \sum_{i=1}^{k} \frac{1}{r\left(C_{i}\right)} \geqq \frac{1}{r(C)}
$$

with equality if, and only if $k=6, r(C)=r\left(C_{1}\right)=\ldots=r\left(C_{k}\right)$.

A graph $G=(V, E)$ is called locally finite if every $x \in V$ has only finitely many neighbours in $G$, i.e., $\left|\Gamma_{G}(x)\right|<\infty$. We need the following general result on convex functions:

THEOREM 3. Let $G=(V, E)$ be a locally finite graph, $f: V \rightarrow \mathbf{R}$ a convex function on $G$. Assume further that $x_{0} v_{0} \in E, f\left(x_{0}\right)=0, f\left(y_{0}\right)=1$; and let $V_{i}$ denote the set of those points in $G$ which can be reached from $y_{0}$ by $a$ monotone increasing path of length at most $i$, i.e.,

$$
V_{i}:=\left\{y \in V \mid \begin{array}{c}
\exists y_{1}, \ldots, y_{j-1} \in V \text { such that } j \leqq i, \\
y_{0} y_{1}, y_{1} y_{2}, \ldots, y_{j-1} y \in E \text { and } \\
f\left(y_{0}\right) \leqq f\left(y_{1}\right) \leqq \ldots \leqq f(y)
\end{array}\right\} .
$$

Then, for every natural number $n$, we have

(1) $\max _{x \in V_{n}} f(x) \geqq 1+\sum_{i=1}^{n} \frac{1}{\left|E_{i}\right|}$,

where

$$
E_{i}:=\left\{u v \in E \mid u \in V_{i-1}, v \in V_{i} \backslash V_{i-1}, f(u) \leqq f(v)\right\}, \quad 1 \leqq i \leqq n .
$$

We close this section by showing how Theorem 1 follows from the last two results.

Let $\mathscr{C}$ be a 6-neighboured circle packing, $G_{\mathscr{C}}=\left(V_{\mathscr{C}}, E_{\mathscr{C}}\right)$ the corresponding graph defined above. If $\mathscr{C}$ is the regular circle packing then there is nothing to prove. Hence, we may suppose without loss of generality that there are two tangent discs $C^{\prime}, C^{\prime \prime} \in \mathscr{C}=V_{\mathscr{C}}$ such that $r\left(C^{\prime}\right)>r\left(C^{\prime \prime}\right)$. Our Theorem 2 implies that

$$
f(C):=\left(\frac{1}{r(C)}-\frac{1}{r\left(C^{\prime}\right)}\right) /\left(\frac{1}{r\left(C^{\prime \prime}\right)}-\frac{1}{r\left(C^{\prime}\right)}\right), \quad C \in \mathscr{C}
$$

is a convex function on $G_{\mathscr{C}}$ which meets the conditions of Theorem 3 with $x_{0}=C^{\prime}, y_{0}=C^{\prime \prime}$. Thus, for every natural $n$, we get (1). 
Suppose now, in order to obtain a contradiction, that

$$
\inf _{C \in \mathscr{C}} r(C)=\epsilon>0 .
$$

Since all elements of $V_{i}$ are covered by a circle of radius $r\left(C^{\prime \prime}\right) i$ around the centre of $C^{\prime \prime}$, we have

$$
\left|V_{i}\right| \leqq\left(r\left(C^{\prime \prime}\right) / \epsilon\right)^{2} i^{2}, \text { for every } i .
$$

On the other hand, $G_{\mathscr{C}}$ is a planar graph, thus any subgraph of $G_{\mathscr{C}}$ contains at most 3 times more edges than vertices. This yields

(2) $\sum_{j=1}^{i}\left|E_{j}\right| \leqq 3\left|V_{i}\right| \leqq 3\left(\frac{r\left(C^{\prime \prime}\right)}{\epsilon}\right)^{2} i^{2} \quad(\forall i)$.

The following easy technical lemma is a simple special case of Karamata's inequality (cf. [1], [5] ).

LEMMA. Let $e_{1}, \ldots, e_{n}$ and $A$ be positive numbers satisfying

$$
\sum_{j \leqq i} e_{j} \leqq A i^{2} \quad \text { for every } i=1, \ldots, n \text {. }
$$

Then

$$
\sum_{i \leqq n} \frac{1}{e_{i}}>(\log n) /(2 A)
$$

Hence (2) implies

$$
\text { (2') } \sum_{i=1}^{n} \frac{1}{\left|E_{i}\right|} \geqq \frac{\epsilon^{2}}{6 r^{2}\left(C^{\prime \prime}\right)} \log n \text {. }
$$

Putting (1) and $\left(2^{\prime}\right)$ together we obtain

$$
\lim _{n \rightarrow \infty} \max _{x \in V_{n}} f(x)=\infty
$$

which is tantamount to $\inf _{C \in \mathscr{C}} r(C)=0$, a contradiction.

2. Proof of theorem 2. We may assume without loss of generality that $C$ is exactly surrounded by the discs $C_{1}, \ldots, C_{k}$ : i.e., each $C_{i}$ is tangent to $C$, $C_{i-1}$ and $C_{i+1}$ (where the subscripts are taken modulo $k$ ). Let $O$ and $O_{i}$ denote the centers of $C$ and $C_{i}(i=1, \ldots, k)$, respectively, and let $\angle O_{i} O O_{i+1}=\varphi_{i}$. We are going to show that

$$
\text { (3) } \frac{1}{r\left(C_{i}\right)}+\frac{1}{r\left(C_{i+1}\right)} \geqq \frac{1}{r(C)}\left(\tan \frac{\varphi_{i}}{4}+\cot \frac{\varphi_{i}}{4}-2\right), \quad i=1, \ldots, k \text {. }
$$

Let us have a closer look e.g. at the discs $C, C_{1}$ and $C_{2}$. Let $P$ denote the centre of the circle $D$ inscribed in $\triangle O O_{1} O_{2}$, and put $\angle O O_{1} P=\alpha$, $\angle \mathrm{OO}_{2} \mathrm{P}=\beta$. Then we have 


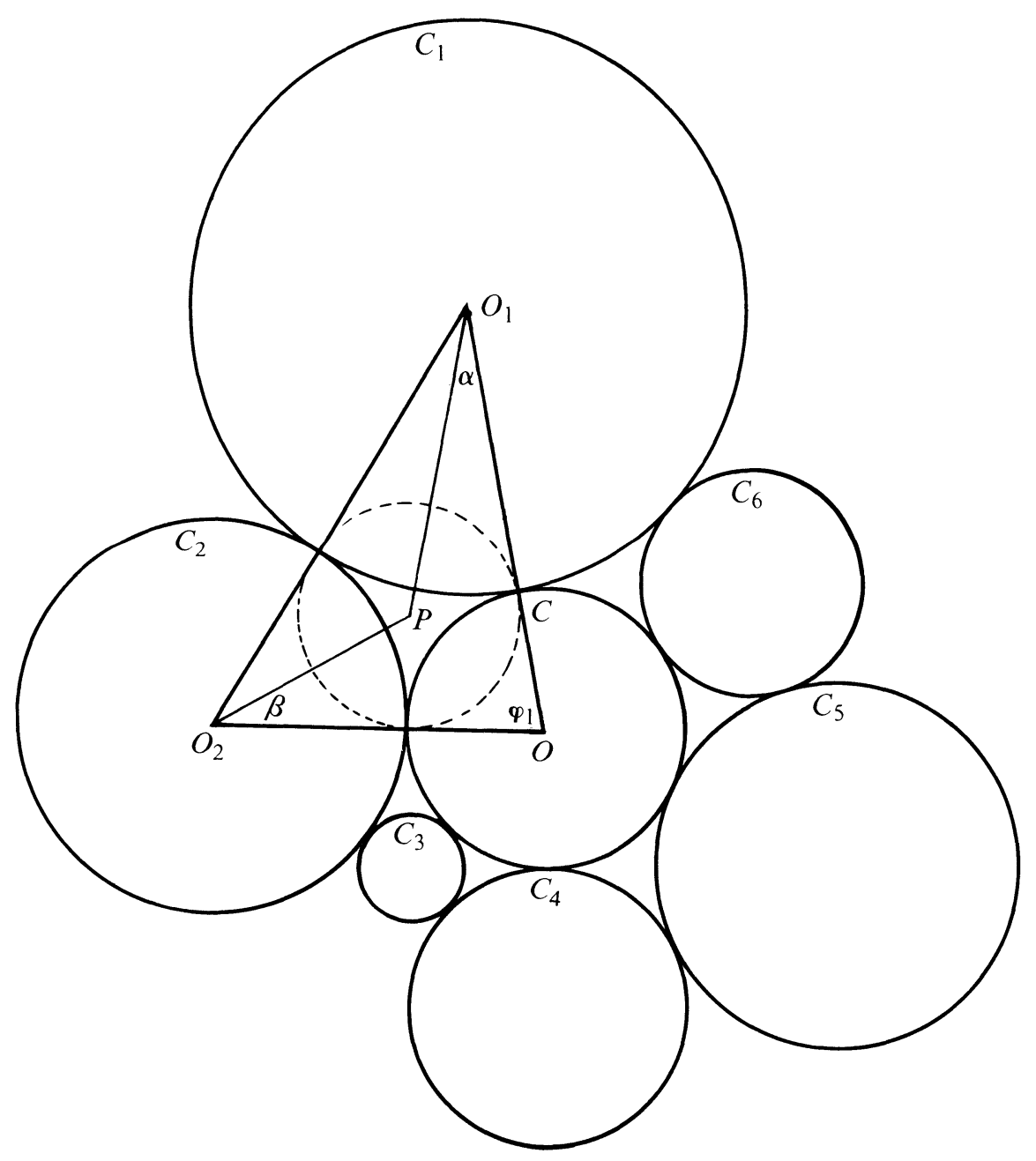

Figure 1 


$$
\begin{aligned}
\frac{r(D)}{r\left(C_{1}\right)}+\frac{r(D)}{r\left(C_{2}\right)} & =\tan \alpha+\tan \beta \geqq 2 \tan \frac{\alpha+\beta}{2} \\
& =2 \tan \frac{\pi-\varphi_{1}}{4}=\tan \frac{\varphi_{1}}{2} \cdot \frac{\left(1-\tan \frac{\varphi_{1}}{4}\right)^{2}}{\tan \frac{\varphi_{1}}{4}} \\
& =\frac{r(D)}{r(C)}\left(\tan \frac{\varphi_{1}}{4}+\cot \frac{\varphi_{1}}{4}-2\right),
\end{aligned}
$$

as required.

Summing up (3) for $i=1, \ldots, k$ and using Jensen's inequality, we obtain

$$
\begin{aligned}
2 \sum_{i=1}^{k} \frac{1}{r\left(C_{i}\right)} & \geqq \frac{1}{r(C)} \sum_{i=1}^{k}\left(\tan \frac{\varphi_{i}}{4}+\cot \frac{\varphi_{i}}{4}-2\right) \\
& \geqq \frac{k}{r(C)}\left(\tan \frac{\pi}{2 k}+\cot \frac{\pi}{2 k}-2\right) \geqq \frac{2 k}{r(C)},
\end{aligned}
$$

for $k \geqq 6$. This completes the proof.

3. Proof of theorem 3. Throughout this section $n$ will be kept fixed,

$$
M:=\max _{x \in V_{n}} f(x) .
$$

Define a subgraph $G^{\prime}=\left(V^{\prime}, E^{\prime}\right) \subseteq G$ by

$$
\begin{aligned}
& V^{\prime}:=V_{n} \cup\left\{x_{0}\right\} \\
& E^{\prime}:\left\{u v \in E \mid \quad u, v \in V_{n}\right\} \cup\left\{x_{0} y_{0}\right\} .
\end{aligned}
$$

We turn $G^{\prime}$ into a digraph $\vec{G}^{\prime}=\left(V^{\prime}, \vec{E}^{\prime}\right)$ by supplying its edges with the following orientation: (a) If $u v \in E^{\prime}$ and $f(u)<f(v)$, then let $\vec{u} v \in \vec{E}$. (b) Every edge belonging to $E_{i} \subseteq E^{\prime}$ will be directed from $V_{i-1}$ to $V_{i}, i=$ $1, \ldots, n$. (c) Any further edge $u v \in E^{\prime}$ (with $f(u)=f(v)$ ) can be directed arbitrarily.

Note that if $u v \in E^{\prime}$ for some $u \in V_{i-2}$ and $v \in V_{i} \backslash V_{i-1}$, then $f(u)$ is necessarily larger than $f(v)$, consequently $u v \notin E_{i}$, and $\vec{v} u \in \vec{E}^{\prime}$.

Let $\mathscr{F}$ denote the class of all functions $\varphi: V^{\prime} \rightarrow \mathbf{R}$ satisfying the following three conditions:

(i) $\varphi\left(x_{0}\right)=0$.

(ii) $f(x) \leqq \varphi(x) \leqq M$ for every $x \in V^{\prime}$.

(iii) There exists a digraph $\vec{G}^{\prime \prime}=\left(V^{\prime}, \vec{E}^{\prime \prime}\right) \subseteq \vec{G}^{\prime}$ such that $\vec{x}_{0} \mathrm{y}_{0}$

$\in \vec{E}^{\prime \prime} \subseteq \vec{E}^{\prime}, \varphi(u) \leqq \varphi(v)$ for every $\vec{u} v \in \vec{E}^{\prime \prime}$, and 
(4)

$$
\frac{1}{\left|\Gamma_{G^{\prime \prime}}(x)\right|} \sum_{y \in \Gamma_{G^{\prime \prime}}(x)} \varphi(y) \geqq \varphi(x) \text { for every } x \in V_{n-1} .
$$

Here $G^{\prime \prime}$ denotes the graph obtained from $\vec{G}^{\prime \prime}$ by ignoring the orientation of the edges, and $\Gamma_{G^{\prime \prime}}(x)$ is, as usual, the set of neighbours of $x$ in $G^{\prime \prime}$.

We clearly have $f \in \mathscr{F}$. To see this, it suffices to show that $\varphi=f$ satisfies (iii) with $\vec{G}^{\prime \prime}:=\vec{G}^{\prime}$. But this follows immediately from the fact that, if $x \in$ $V_{n-1}$, then $f(y)<f(x)$ for every $y \in \Gamma_{G}(x) \backslash \Gamma_{G^{\prime}}(x)$.

Let $f_{0}$ be a maximal element of $\mathscr{F}$, and let $\vec{G}_{0}^{\prime \prime}=\left(V^{\prime}, \vec{E}_{0}^{\prime \prime}\right)$ denote the corresponding digraph in (iii). (The existence of a maximal element is obvious, e.g. by compactness arguments.) Next we prove that $f_{0}$ satisfies (4) with equality at every (non-isolated) vertex $x \in V_{n-1}$. That is,

(5) $\frac{1}{\left|\Gamma_{G_{0}^{\prime \prime}}(x)\right|} \sum_{y \in \Gamma_{G_{0}^{\prime \prime}}(x)} f_{0}(y)=f(x) \quad\left(x \in V_{n-1}, \Gamma_{G_{0}^{\prime \prime}}(x) \neq \emptyset\right)$.

Assume indirectly that, for some $x \in V_{n-1}$, the left-hand side of (5) is strictly larger than the right-hand side. Set

$$
\begin{aligned}
& \vec{F}:=\left\{\vec{x} z \in \vec{E}_{0}^{\prime \prime} \mid f_{0}(x)=f_{0}(z)\right\}, \\
& f_{\epsilon}(t):= \begin{cases}f_{0}(t) & \text { if } t \neq x \\
f_{0}(x)+\epsilon & \text { if } t=x .\end{cases}
\end{aligned}
$$

If $\epsilon>0$ is sufficiently small, then $f_{\epsilon}$ and $\vec{G}_{\epsilon}^{\prime \prime}:=\left(V^{\prime}, \vec{E}_{0}^{\prime \prime} \backslash \vec{F}\right)$ meet all the conditions (i), (ii) and (iii), contradicting the maximum property of $f_{0}$.

Now let $\vec{f}_{0}(\vec{x} y):=f_{0}(y)-f_{0}(x)$ for every $\vec{x} y \in \vec{E}_{0}^{\prime \prime}$, and observe that (5) is equivalent to

$$
\sum_{\vec{x}, \in \vec{E}_{0}^{\prime \prime}} \tilde{f}_{0}(\vec{x} y)=\sum_{\vec{v} x \in \vec{E}_{0}^{\prime \prime}} \tilde{f}_{0}(\vec{v} x) \quad\left(\forall x \in V_{n-1}\right) .
$$

Hence, in another terminology we can say that $\vec{G}_{0}^{\prime \prime}$ is a network (with one source $x_{0}$ and some sinks in $\left.V_{n} \backslash V_{n-1}\right)$, and assigning the value $\widehat{f}_{0}(\vec{u} v)$ $\geqq 0$ to each edge $\vec{u} v \in \vec{E}_{0}^{\prime \prime}$ we obtain a flow. (Note that there can not be any source in $V_{n} \backslash V_{n-1}$, because $f_{0}(x)=M$ for every $x \in V_{n} \backslash V_{n-1}$.)

Since the flow value disappearing at the sinks is equal to the value entering our network, we have

$$
\sum_{y \in V_{n} \backslash V_{n-1}} \sum_{x \vec{y} \in \vec{E}_{0}^{\prime \prime}} f_{0}(\vec{x} y)=\vec{f}_{0}\left(\vec{x}_{0} y_{0}\right)=f_{0}\left(y_{0}\right) .
$$

Further, it is obvious that every edge of positive flow value leaving $V_{i-1}$ belongs to $E_{i}$, thus

$$
\sum_{\substack{x y \in \vec{E}_{0}^{\prime} \\ x y \in E_{i}}} \vec{f}_{0}(\vec{x}) \geqq \mathcal{f}_{0}\left(\vec{x}_{0} y_{0}\right)=f_{0}\left(y_{0}\right), \quad i=1, \ldots, n .
$$


Hence it follows by (6) that

$$
\begin{aligned}
\sum_{x y \in E_{0}^{\prime \prime}}\left(f_{0}(y)-f_{0}(x)\right)^{2} & =\sum_{y \in V^{\prime}} f_{0}(y) \sum_{x \in \Gamma_{G_{0}^{\prime \prime}}(y)}\left(f_{0}(y)-f_{0}(x)\right) \\
& =\sum_{y \in V_{n} \backslash V_{n}: 1} f_{0}(y) \sum_{x \in \Gamma_{G^{\prime}(y)}(y)}\left(f_{0}(y)-f_{0}(x)\right) \\
& =\sum_{v \in V_{n} \backslash V_{n},} M \sum_{\vec{x} v \in \vec{E}_{0}^{\prime \prime}} \tilde{f}_{0}(\vec{x} y)=M f_{0}\left(y_{0}\right) .
\end{aligned}
$$

On the other hand, applying (7) and Jensen's inequality, we obtain

$$
\begin{aligned}
\sum_{x y \in E_{0}^{\prime \prime}}\left(f_{0}(y)-f_{0}(x)\right)^{2} & \geqq\left(f_{0}\left(y_{0}\right)-f_{0}\left(x_{0}\right)\right)^{2}+\sum_{i=1}^{n} \sum_{\substack{x y \in \vec{E}_{0}^{\prime \prime} \\
x y \in E_{i}}} \vec{f}_{0}^{2}(\vec{x} y) \\
& \geqq f_{0}^{2}\left(y_{0}\right)+\sum_{i=1}^{n} \frac{f_{0}^{2}\left(y_{0}\right)}{\left|E_{0}^{\prime \prime} \cap E_{i}\right|} \\
& \geqq f_{0}^{2}\left(y_{0}\right)\left(1+\sum_{i=1}^{n} \frac{1}{\left|E_{i}\right|}\right) .
\end{aligned}
$$

Now using the fact that $f_{0}\left(y_{0}\right) \geqq f\left(y_{0}\right)=1$, (1) immediately follows. This completes the proof of Theorem 3 .

4. Remarks and acknowledgement. We would like to express our gratitude to L. Fejes Tóth and N. Dolbilin for their valuable comments and suggestions.

Special thanks are due to I. Vincze who has considerably simplified our first proof of Theorem 2. Our original argument, however, established also the following result:

TheOREM 4. Let $C, C_{1}, \ldots, C_{k}(k \geqq 3)$ be non-overlapping discs in the plane, and suppose that $C$ is exactly surrounded by $C_{1}, \ldots, C_{k}$ (see Section 2). Further, let $|n| \geqq 100$. Then

$$
\frac{1}{k} \sum_{i=1}^{k} r^{n}\left(C_{i}\right) \geqq\left(\frac{\sin (\pi / k)}{1-\sin (\pi / k)} r(C)\right)^{n},
$$

with equality if and only if $r\left(C_{1}\right)=\ldots=r\left(C_{k}\right)$. 
The proof can be omitted here, since L. Fejes Tóth [4] has recently managed to prove the same assertion for all $n \geqq 1$.

Added in proof. We thank Adrian Bondy for calling our attention to a result of C. St. J. A. Nash-Williams: Random walk and electric current in networks (Proc. Cambridge Philos. Soc. 55 (1959), 181-199) which is closely related to our Theorem 3 .

\section{REFERENCES}

1. E. F. Beckenbach and R. Bellman, Inequalities (Springer, Berlin-Göttingen-Heidelberg, 1961).

2. E. B. Dynkin and A. A. Juschkewitsch, Sätze und Aufgaben über Markoffsche Prozesse (Springer, Berlin-Heidelberg-New York, 1969).

3. L. Fejes Tóth, Research problem, Periodica Math. Hung. 8 (1977), 103-104.

4. Compact packing of circles, Studia Sci. Math Hungar. (to appear).

5. J. Karamata, Sur une inégalité relative aux fonctions convexes, Publ. Math. Univ. Belgrade $l$ (1932), 145-148.

6. K. A. Rybnikov, Problems of combinatorial analysis (in Russian) (MIR Publ. Co., Moscow, 1980).

Hungarian Academy of Sciences, Budapest, Hungary 